\title{
Photoanisotropy in polarization- sensitive polymer materials based on the media with covalently-bonded components
}

Irakli Chaganava, Barbara Kilosanidze, George Kakauridze, Luis Oriol, Milagros Piñol, et al.

Irakli Chaganava, Barbara Kilosanidze, George Kakauridze, Luis Oriol, Milagros Piñol, Alfonso Martinez-Felipe, "Photoanisotropy in polarizationsensitive polymer materials based on the media with covalently-bonded components," Proc. SPIE 10101, Organic Photonic Materials and Devices XIX, 101010T (16 February 2017); doi: 10.1117/12.2249997 


\title{
Photoanisotropy in polarization-sensitive polymer materials based on the media with covalently-bonded components
}

\author{
Irakli Chaganava $^{{ }^{*}}$, Barbara Kilosanidze ${ }^{1}$, George Kakauridze ${ }^{1}$, \\ Luis Oriol$^{2}$, Milagros Piñol ${ }^{2}$, Alfonso Martinez-Felipe ${ }^{2}$ \\ 1 - Laboratory of Holographic Recording and Processing of Information, Institute of Cybernetics \\ at the Georgian Technical Univ., 5 Sandro Euli Str., 0186 Tbilisi, Georgia; \\ 2 - Institute of Materials Science of Aragon (ICMA), University of Zaragoza - CSIC, \\ Faculty of Science. Pedro Cerbuna 12, 50009, Zaragoza, Spain.
}

\begin{abstract}
The well-known scalar photochromism phenomenon is a reversible phototransformation of chemical species between two forms having different absorption spectra. It is observed under the action of actinic light regardless of its polarization state. Unlike this in some high-efficient polarization-sensitive azopolymeric materials, we have observed a welldeveloped vector polyphotochromism which appears as a light-induced area with spectral selectivity for the linearly polarized probing beams. A sharp change in the transmission spectrum of the material have been observed when we placed an irradiated area of the sample between crossed polarizers, while the transmission spectrum of the sample remained practically unchanged in case of probing by unpolarized light. The effect has a purely vector nature, while the transmission spectrum of the exposed material essentially changes in case of observing between crossed polarizers and the change in the spectrum unambiguously depends on the energy exposure. A significant dependence of the kinetic of the vector polyphotochromism induction on the power density of linearly polarized actinic light $(445 \mathrm{~nm})$ is shown for probing beam of $635 \mathrm{~nm}$. It is also shown that the kinetics of the effect depends on the photosensitive layer thickness and the concentration of the chromophore. The experiments were carried out for two synthesized side-chain azopolymers obtained as immobilized polar azo dyes on polymethylmethacrylate backbone. It is clearly shown a light-controlled spectral selectivity of the sample activated by the various doses of the stimulating radiation.
\end{abstract}

Keywords: vector polyphotochromism, interference gutchimg, photoelasticity, polarization-sensitive material, photoanisotropy, light-manipulating organic material, side-chain azopolymer

\section{INTRODUCTION}

As is well known scalar photochromism is a reversible phototransformation of a chemical structure between two forms having different absorption spectra. It is observed under the action of actinic light regardless of its polarization state. This property is widely used in science and engineering. This phenomenon is well studied and a large number of papers are devoted both to the study of this phenomenon and to its applications. ${ }^{1-3}$

It is known that in conventional polarization-sensitive materials photoanisotropy is induced under the action of actinic polarized light. It reaches its maximum and then remains unchanged even after long exposure, remaining at a level of saturation, during which there is the maximum transmission of the probe beam (Figure 1, curve 1). ${ }^{4-10}$

Unlike this in some high-efficient polarization-sensitive materials obtained by us, we have observed the anomalous kinetics of photoanisotropy induction after reaching its maximum value when material is exposed by actinic linearly polarized radiation and simultaneously is probed by monochromatic nonactinic linearly polarized light beam. ${ }^{11-13}$

After reaching the maximum the intensity of the transmitted probing beam starts decreasing with radiant exposure of inducing light increasing and almost reaches zero for the given wavelength of the probing beam (Figure 1, curve 2). The intensity of the probing beam passing through the exposed material essentially depends on radiant exposure of the inducing light, on a wavelength of the probing beam and on the thickness of the emulsion layer.

*irakli@sportuni.ge; phone+995 598 104539; www.irakli.info

Organic Photonic Materials and Devices XIX, edited by Christopher E. Tabor, François Kajzar,

Toshikuni Kaino, Yasuhiro Koike, Proc. of SPIE Vol. 10101, 101010T · C 2017 SPIE

CCC code: $0277-786 \mathrm{X} / 17 / \$ 18 \cdot$ doi: $10.1117 / 12.2249997$ 


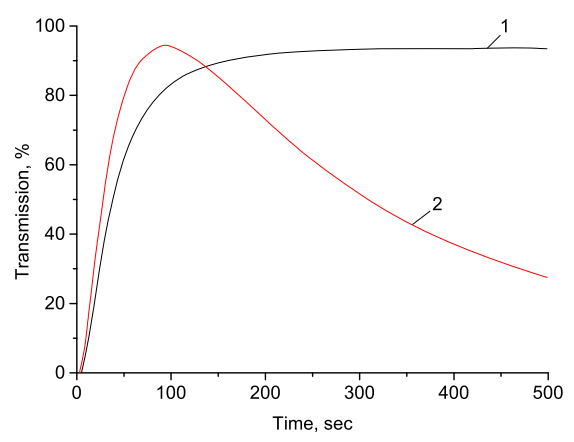

Figure 1. Photoanisotropy induction kinetic curves:

1 - usual course; 2 - unusual course

It was shown ${ }^{11-13}$ that this phenomenon has the purely vector nature because the transmission of the material during probing by nonpolarized light is practically unchanged at different radiant exposures. However, an essential change in transmission was observed under probing the sample by linearly polarized nonactinic light. This phenomenon can be observed between crossed polarizers. It has been shown ${ }^{11}$ that this phenomenon has an interference nature and it is related to the increase in path difference of the ordinary and extraordinary rays when the probing beam passes through the sample.

In this paper the results of the further investigation of this phenomenon in side-chain azopolymers are presented and the kinetic curves of transmission are shown, which were obtained for two wavelengths of probing beam (532 nm and 635 $\mathrm{nm})$ and for different exposure with linearly polarized light, actinic for material under investigation $(445 \mathrm{~nm})$ and for different power densities of the inducing beam as well as on the photosensitive layer thickness. The mechanism of the phenomenon is discussed.

\section{PHOTOMETRIC MEASUREMENTS}

For quantitative description of anisotropy induced by polarized light the method of description photoinduced anisotropy is based on the characteristic which can be obtained by one measurement. This parameter was introduced and investigated in Ref. 14 and was called the effective anisotropy of the medium.

Let the anisotropic medium is described by the Jones matrix ${ }^{15}$

$$
M=\left(\begin{array}{cc}
\exp \left(-i \kappa d \hat{n}_{1}\right) & 0 \\
0 & \exp \left(-i \kappa d \hat{n}_{2}\right)
\end{array}\right) .
$$

Here $\hat{n}_{1}$ and $\hat{n}_{2}$ are complex refractive indexes of the medium, $d$ is the thickness of the medium, $\kappa=2 \pi / \lambda, \lambda$ is a wavelenghth of the inducing light.

The module of the anisotropic invariant of the matrix (1), which was called effective photoanisotropy of the medium, is presented as ${ }^{14}$

$$
\left.A_{e f f}=\frac{1}{4} \mid \exp \left(-i \kappa d \hat{n}_{1}\right)-\exp \left(-i \kappa d \hat{n}_{2}\right)^{2}=\frac{1}{2} \exp [-2 \kappa d(\overline{n \tau})] \operatorname{ch} \kappa d \Delta(n \tau)-\cos \kappa d \Delta n\right],
$$

where $(\overline{n \tau})=\frac{1}{2}\left[(n \tau)_{1}+(n \tau)_{2}\right]$ is the average absorption coefficient, $\Delta(n \tau)$ and $\Delta n$ characterize the anisotropy of absorption and birefringence respectively.

From (2) we can see that the value of effective anisotropy is defined by joint contribution of anisotropic characteristics of the material (birefringence and anisotropy of absorption) and its transmission. The analysis of the expression (2) shows that with the growth of $\Delta n$ and $\Delta(n \tau)$ a multiplier in brackets taking into account the summary contribution of birefringence and anisotropic absorption. This parameter allows different light-sensitive materials to be described and to be compared by a simple way. 
If probing is carried out by the light beam with a wavelength outside the absorption band then we obtain from (2)

$$
A_{\text {eff }}==\frac{1}{2}[1-\cos \kappa d \Delta n]
$$

It follows from (3) that

$$
\Delta n=\frac{1}{\kappa d} \arccos \left(1-2 A_{e f f}\right)+m \pi,
$$

where $m=0,1,2, \ldots$ in the maximum and minimum points of the kinetic curve (however, we have fractional values of $m$ at intermediate points of the kinetic curves corresponding to the values of $A_{\text {eff }}$ for this radiant exposure).

For the experimental measurement of effective anisotropy in ${ }^{14}$ it was shown that $A_{e f f}$ is equal to the transmission of anisotropic medium placed between crossed polarizers at an angle between the axis of anisotropy and the axis of one of the polarizers equal to $45^{0}$ and it is measured in units of intensity. But it is convenient to measure this value in the experiment in \% relative to the transmission of the unradiated sample placed between parallel polarizers.

To study the properties of the polarization-sensitive materials and kinetic of photoanisotropy induction in them under the action of polarized actinic light, we used the laboratory photometric set-up, the optical scheme of which is shown in Figure 2.

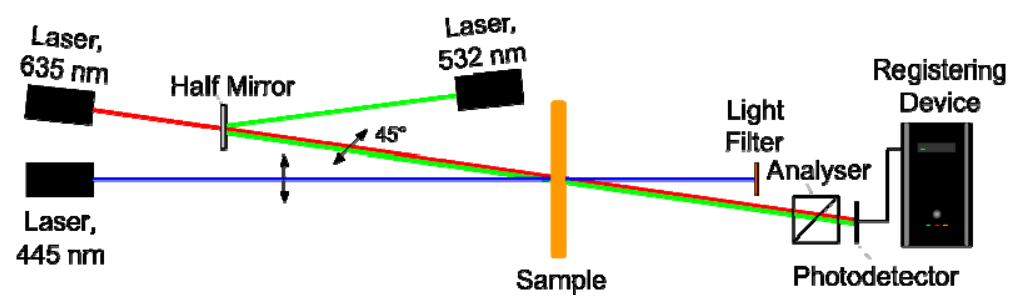

Figure 2. Experimental setup for investigation of the photoanisotrpy kinetics.

In the experimental scheme we used DPSS laser emitting the light with a wavelength of $445 \mathrm{~nm}$ which is actinic for all the researched materials as they have a reasonable spectral absorption on that wavelength to induce the essential phototransformation of the azodye chromophore component molecules. As a probing beam we used laser at a wavelength of $532 \mathrm{~nm}$ and $635 \mathrm{~nm}$.

A linearly polarized actinic laser beam induced the photoanisotropy in the investigated samples and then was blocked behind the sample by an appropriate filter. Simultaneously the probing beam passed the sample through the photoactivated area. This beam is linearly polarized at an angle of 45 degrees to the polarization of the inducing beam. The analyzer is oriented orthogonally to the polarization of the probing beam. In case of the absence of photoanisotropy (if the sample is isotropic) the probing beam did not pass through the analyzer. In case of optical anisotropy induction, the axis of which corresponds to the direction of the polarization vector of the inducing beam (that is, makes 45 degrees with the polarization vector of the probing beam) some portion of the intensity of the probing beam passes through the analyzer and this intensity unambiguously depends on the value of inducing photoanisotropy. Note that both the wavelengths of probing $(532$ and $635 \mathrm{~nm}$ ) are far away from the absorption band of the polarization-sensitivity material. In this case linear birefringence has a major role and dichroism can be neglected.

For some samples of high-efficiency polarization-sensitive material the anomalous behavior of kinetic curve for $A_{\text {eff }}$ (Figure 1, curve-2) have obtained. The value of $A_{\text {eff }}$ reached nearly zero at high exposures. Thus, we have supposed that this phenomenon is related to the interference quenching of probing beam, which occurs as a result of the path difference between the ordinary and extraordinary rays when the probing beam passes through the exposed sample. ${ }^{11}$ The value of the path difference depends on the photoelastic coefficient of the material and the thickness of the photosensitive layer for given exposure. As a result of the selective absorption of a quantum of inducing linearly polarized light the chromophore molecule undergoes trans-cis isomerization, which in turn leads to the appearance of ordered microstrains in polymer matrix of the material. And since the polymer matrix possesses photoelasticity, then the ordered microstrains 
result in macroscopically observed photoanisotropy. When reach a certain level of photoanisotropy for which the birefringence coefficient $\Delta n$ reaches such magnitude that the path difference of the ordinary and extraordinary rays of the probing beam equals to half of the wavelength of this beam, in this case the transmission of the probing beam for given wavelength will be maximum. A further increase in exposure leads to increase in $\Delta n$ and, accordingly, to increase in the path difference, which in turn results in interference quenching and in case the path difference is equals to wavelength the probing beam is totally quenched. ${ }^{11}$ It must be emphasized that in this case the value of photoanisotropy is actually increased although the intensity of the probing beam is reduced.

\section{VECTOR POLYPHOTOCHROMISM}

In the process of the investigation the properties of high-efficient polarization-sensitive materials, we have observed the unusual course of the kinetic curve of the probing beam transmission intensity change (Figure 1, curve 2) in some materials during an exposure them by linearly polarized actinic radiation and simultaneous probing by monochromatic nonactinic linearly polarized light beams. The result of an interaction of light with the observed materials depends on the probing light wavelength and on the actinic light exposure rate. The preliminary results of the investigation of this phenomenon were already presented. ${ }^{11-13}$

In Ref. [12,13] we used the high-efficient polarization-sensitive material which is a solid solution by its compositions based on MPY-NH $\mathrm{NH}_{4}$ the ammonium salt of the polyhydric acid azodye Mordant Pure Yellow [also known as Mordant Yellow 26 (MY-26)] in the matrix of photogelatin. We used the samples with the thickness about $20 \mu \mathrm{m}$. The absorbance spectra of this kind of material were obtained in polarized light for different exposures from 0 to $250 \mathrm{~J} / \mathrm{cm}^{2}$ and a series of experimental spectral curves was obtained and converted to the transmission curves subsequently (Figure 4) and at the same time, the transmission spectra in unpolarized light differ insignificantly for different radiant exposures (Figure 3 ).

\begin{tabular}{|c|c|c|c|c|c|c|c|c|c|c|c|}
\hline EXPOSITION & H0 & H1 & H2 & H3 & H4 & H5 & H6 & H7 & H8 & H9 & H10 \\
\hline $\mathrm{J} / \mathrm{cm}^{2}$ & 0 & 30 & 60 & 80 & 86 & 94 & 106 & 130 & 170 & 215 & 250 \\
\hline
\end{tabular}

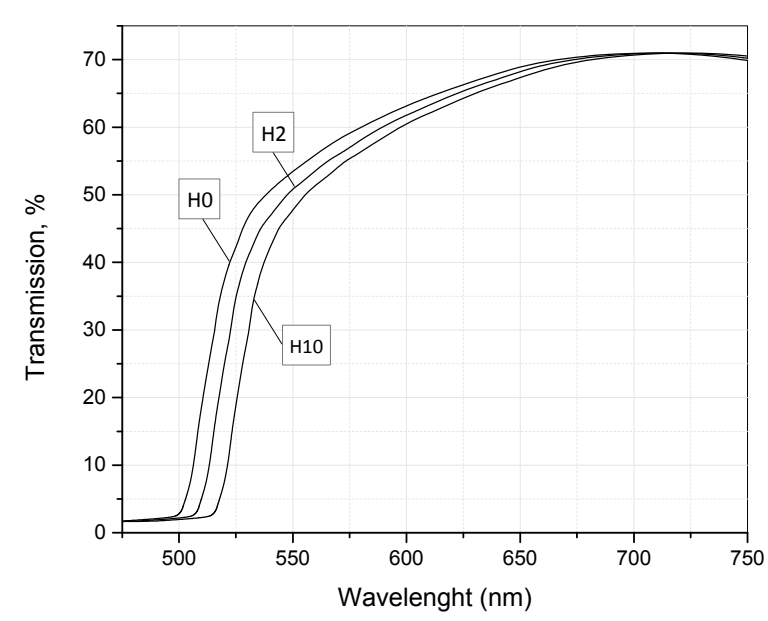

Figure 3. Transmission spectra of the sample material in case of unpolarized probing beams for the different actinic light exposures from 0 up to $250 \mathrm{~J} / \mathrm{cm}^{2}$

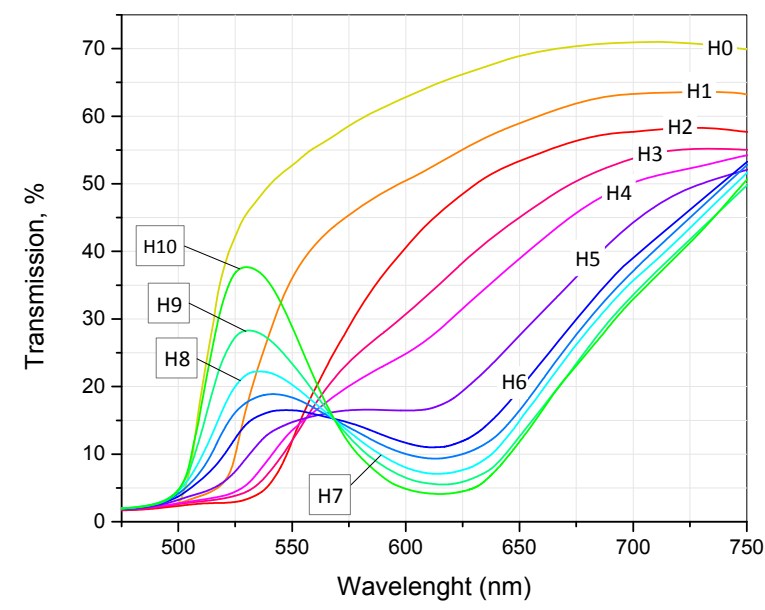

Figure 4. Transmission spectra of the sample material in case of linearly polarized probing beams for the different actinic light exposures from 0 up to $250 \mathrm{~J} / \mathrm{cm}^{2}$

In our recently conducted study, we identified several conditions enhance the described light-induced effect, one of the most influential which turned out to be obvious is the degree of a mutual integration of the material component molecules. ${ }^{12}$ To increase the level of integration of the material components on the molecular level we have synthesized a side-chain type azopolymers, ${ }^{16,17}$ which are immobilized azodyes 4-[4-(trifluoromethyl)phenyl]phenol and 4-[(4- 
hydroxyphenyl)diazenyl]benzonitrile on polymethylmethacrylate (Figure 5, Formula B). In these azopolymers the chromophores are linked to the polymer via covalent bond.

Material-1
$\mathrm{R}=\mathrm{CF}_{3}$

Figure 5. Formula A - photosensitive composed material: chromophoric component (a1) and polymeric component (a2); Formula B - integrated material: polymethylmethacrylate azopolymer containing azodye R-phenylazophenol in side chain in molar proportion of $\mathrm{n}=1 ; \mathrm{m}=7$. Where in Material-1 and Material-2 radical $\mathrm{R}=\mathrm{CF}_{3}$ and in Material-3 and Material-4 radical $\mathrm{R}=\mathrm{CN}$.

We have studied the kinetics of the probing beam transmission through the exposing area of the synthesized materials (Figure 6):
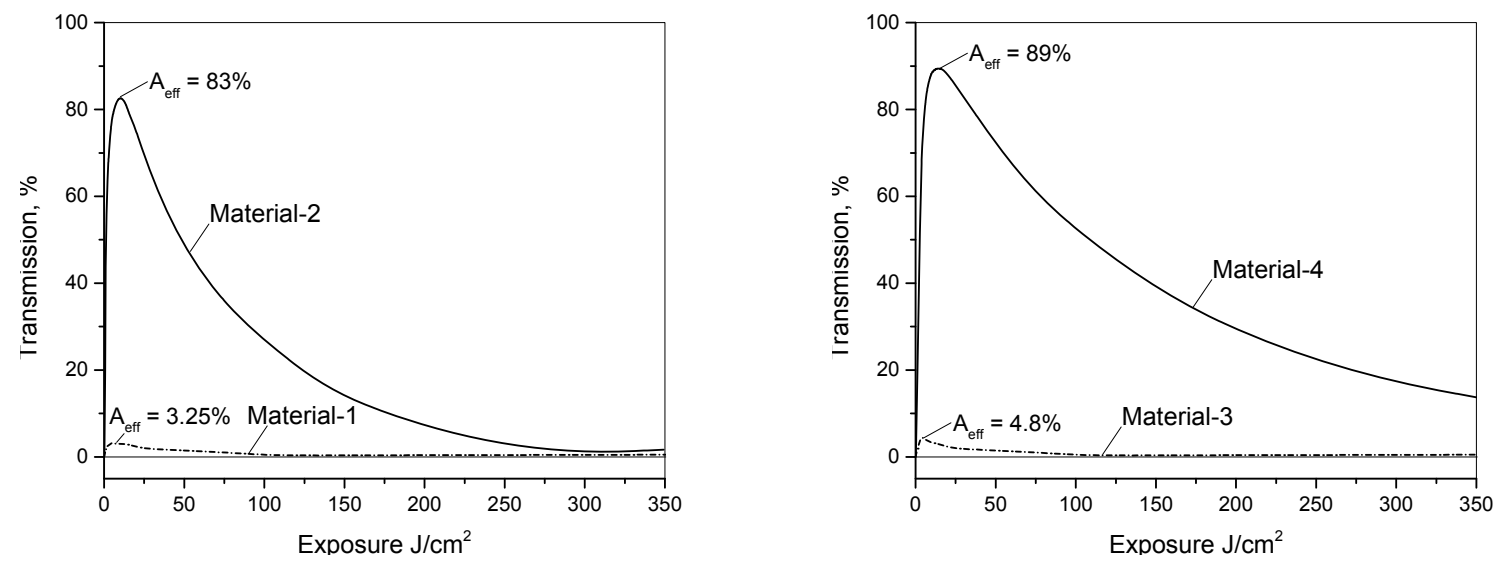

Figure 6. Kinetics of the probe beam transmission through the researched materials (see Figure 5 for structures): curves Material-1 and Material-3 are the solid solutions of components without covalent binding; curves Material-2 and Material-4 are azopolymers with components connected by covalent bonds (for $635 \mathrm{~nm}$ probing beam). The film thickness of the material samples is $15 \mu \mathrm{m}$.

The thickness of the investigated material samples as well as their optical density was the same. For comparison, in the bottom of Figure 6 there are the kinetic curves of the transmission for solid solutions in methylmethacrylate (without covalent bonds) with the same optical density of actinic light as in said-chain azopolymers. It is seen from Figure 6 that the covalent bond obviously increases the level and speed of achievement high values of photoanisotropy which subsequently results in appearing the vector polyphotochromism effect. In addition, the thickness of the samples has a significant impact on the path difference between the ordinary and extraordinary rays and as a result on the appearance of this effect. The results of the investigation of dependence on the thickness were shown in Ref. [13]. 
The curves of changes in the probing beam intensity when it passes through the sample are found to have a different shape in case of probing beams with different wavelengths. Figure 7 shows the kinetic curves of transmission obtained by means of photometric set-up (Fig. 2) and the corresponding values of birefringence $\Delta n$ induced in the samples of MY-26/GEL and Material-2 obtained by probing beam with wavelengths of $635 \mathrm{~nm}$ and $532 \mathrm{~nm}$. The inducing radiation with a wavelength of $445 \mathrm{~nm}$ and a power density of about $750 \mathrm{~mW} / \mathrm{cm}^{2}$ have been used.
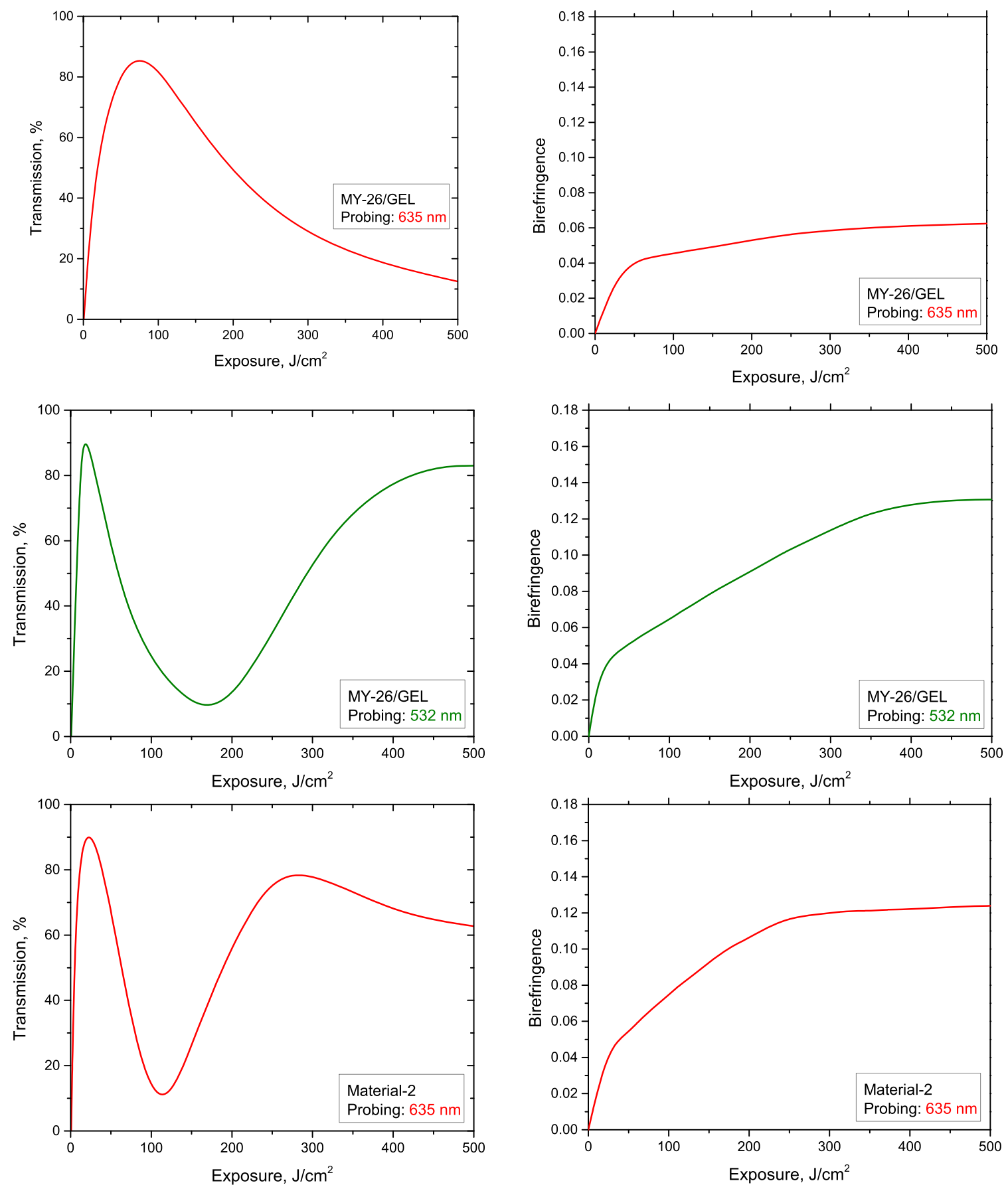

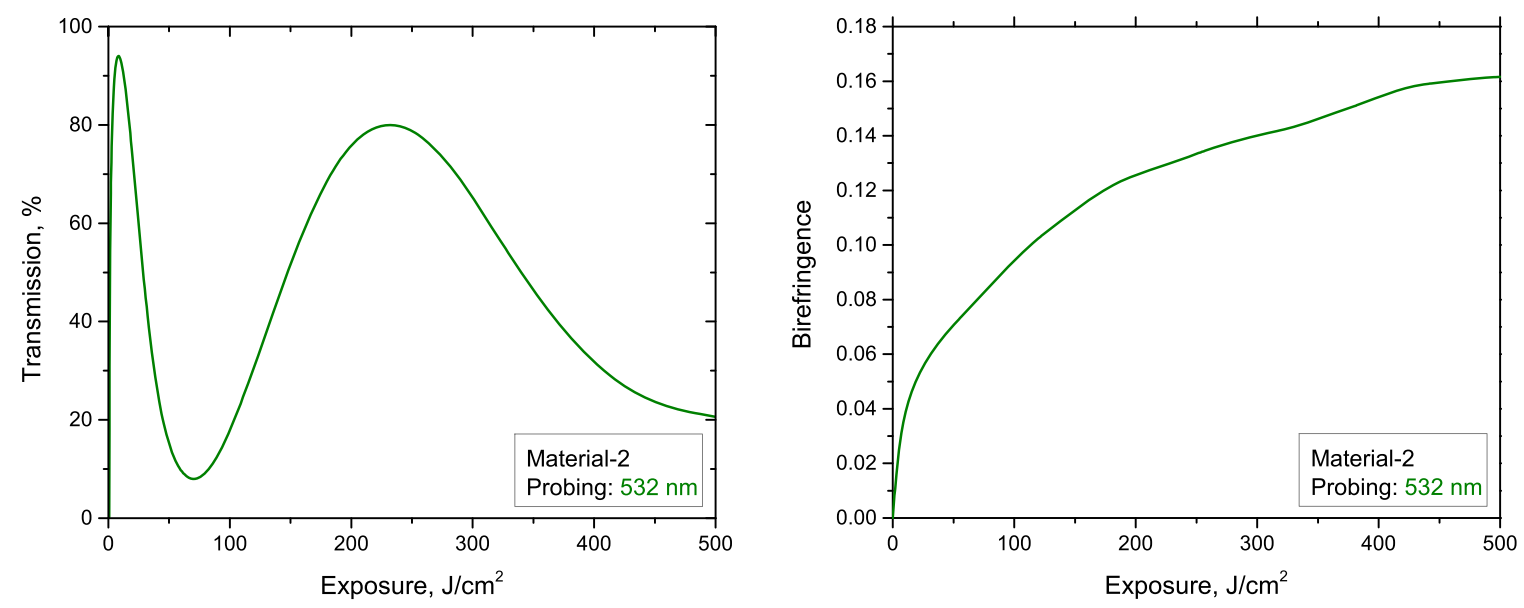

Figure 7. Kinetics of transmission of the probing beam intensity and kinetics of birefringence $\Delta n$ to be induced in a sample of the material based on MY-26/GEL and Material-2 for two wavelengths of the probing beam $635 \mathrm{~nm}$ and 532 $\mathrm{nm}$. Power density of the inducing beam $(445 \mathrm{~nm})$ was about $750 \mathrm{~mW} / \mathrm{cm}^{2}$. The film thickness is $20 \mu \mathrm{m}$.

As can be seen from the curves on Fig. 7, the value of induced photoanisotropy and rate of its rise increases significantly with increasing mutual integration of the polarization-sensitive material's components. For materials with the strongest integration, in the side-chain azopolymers with covalent bonds between the chromophore and polymer matrix molecules the third order of interference of the probing beam is observed in the kinetic of transmission. In this case the maximum value of the induced birefringence reaches 0.162 for the Material-2.

\section{CONCLUSIONS}

We have carried out the further investigation of the phenomenon of vector polyphotochromism. It was shown that the kinetics of the effect depends on the degree of integration of the component of the polarization-sensitive material and the value of induced photoanisotropy and rate of its rise increases significantly with increasing the mutual integration of the polarization-sensitive material components.

Vector polyphotochromism observed in materials in which at least a second order of interference of the ordinary and extraordinary rays is achieved (the path difference is more than $\lambda / 2$ ), and respectively $\Delta n$ is more than 0.04 . In materials with the strongest integration, in the side-chain azopolymers with covalent bonds between the molecules of the chromophore and the polymer matrix the third order of interference of the probing beam is observed in the transmission kinetics and thus the maximum value of the induced birefringence reaches 0.162 .

Vector polyphotochromic effect may be used for creating the spectrally selective dynamic polarization holographic gratings, indicators and displays based on a new physical principle, and also for creating spatial light modulators and dynamic polarization spectral filters controlled by light. Such dynamic gratings in turn can be used in wavelengthdivision multiplexing (WDM) technology for all-optical switches, as well as for creating light-controlled spectral valves.

\section{ACKNOWLEDGEMENTS}

The authors gratefully acknowledge support by the Shota Rustaveli National Science Foundation and Science\& Technology Center in Ukraine (STCU) under grants No. AR/209/6-120/14 and No. 6069 (04/06). 


\section{REFERENCES}

[1] Brown, G. H., ed., [Photochromism] Techniques of Chemistry Vol. III, Wiley-Interscience, New York (1971).

[2] Crano, J. C. and Guglielmetti, R. J., [Organic Photochromic and Thermochromic Compounds], Plenum Press (1999).

[3] ed. by Dürr, H. and Bouas-Laurent, H., [Photochromism: Molecules and Systems: Molecules and Systems], Gulf

Professional Publishing (2003).

[4] Kakichashvili, Sh. [Polarization holography], Nauka, Leningrad (1989).

[5] Kakichashvili, Sh. and Shaverdova, V., "Photoanisotropy in Mordant Pure Yellow azo-dyes," J. Sci. Appl. Photogr. Cinem. (USSR) 24, 342-345 (1979).

[6] Kakichashvili, Sh., Kilosanidze, B. and Shaverdova, V., "Anisotropy and gyrotropy of dye mordant pure-yellow induced by linear polarized light," Opt. Spectrosc. 68, 1309-1312 (1990).

[7] Chaganava, I., Kakauridze, G. and Kilosanidze, B., "Photoanisotropy in polarization-sensitive medium developed on the basis of polar water-soluble components," Proc. SPIE 7957, 7957-14 (2011).

[8] Chaganava, I., Kakauridze, G., Kilosanidze, B. and Datukishvili, G., "Development of high-performance, stable and moisture-resistant polarization-sensitive materials," Proc. SPIE 8126, 8126-51 (2011).

[9] Hvilsted, S., Andruzzi, F., Kulinna, C., Siesler, H.W. and Ramanujam, P.S., "Novel side-chain crystalline polyester architecture for reversible optical storage," Macromolecules 28, 2172-2183 (1995).

[10] Sekkat, Z. and Dumont, M., "Polarization effects in photoisomerization of azo dyes in polymeric films," Apl. Phys. B53, 121-123 (1991).

[11] Chaganava, I., Kakauridze, G., Kilosanidze, B. and Mshvenieradze, Yu., "Light-controlled vector polyphotochromism," Proc. SPIE 9137, 9137-14 (2014).

[12] Chaganava, I., Kakauridze, G., Kilosanidze, B. and Mshvenieradze, Yu., "Vector photochromism in polarizationsensitive materials," Optics Letters 39, 3841-3844 (2014).

[13] Chaganava, I., Kilosanidze, B. and Kakauridze, G., "Light manipulating vector polyphotochromatic behavior in polarization-sensitive materials", Proc. SPIE 9564, Light Manipulating Organic Materials and Devices II, 95640L (2015).

[14] Balabanov, A., Kakauridze, G., Kakichashvili, Sh., Savitski, A., Shaverdova, V. and Shvaitser, Ya ., "On the investigation of spectral characteristics of photoanisotropy," Optics and Spectroscopy, 67, 409-412 (1989).

[15] Jones, R.C., "A new calculus for the treatment of optical systems," J. Opt. Soc. Am. 31, 488-493 (1941).

[16] Stähler, K., Selb, J., Barthelemy, P., Pucci, B., and Candau, F., "Novel hydrocarbon and fluorocarbon polymerizable surfactants: synthesis, characterization and mixing behavior," Langmuir, 14(17), 4765-4775 (1998).

[17] del Barrio, J., Tejedor, R. M., Chinelatto, L. S., Sánchez, C., Piñol, M. and Oriol, L. "Bistable mesomorphism and supramolecular stereomutation in chiral liquid crystal azopolymers," Journal of Materials Chemistry, 19(28), 4922- 4930 (2009). 